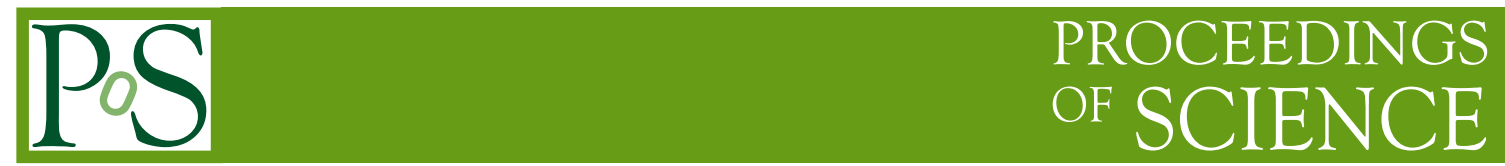

\title{
New insights into soft gluons and gravitons
}

\author{
Chris D. White* \\ University of Glasgow \\ E-mail: Christopher.White@glasgow.ac.uk
}

\begin{abstract}
The study of gluon radiation in QCD, in the limit of small ("soft") momentum, remains an active research area, with a variety of phenomenological and theoretical applications. Soft gluon emission leads to large logarithms in perturbation theory which have to be summed up to all orders in the coupling, and also governs the structure of infrared singularities. Recently, new techniques and mathematical structures have been discovered, which enhance our understanding of these allorder properties. This contribution will review a number of key topics, including the relationship between QCD and gravity.
\end{abstract}

36th International Conference on High Energy Physics,

July 4-11, 2012

Melbourne, Australia

${ }^{*}$ Speaker. 


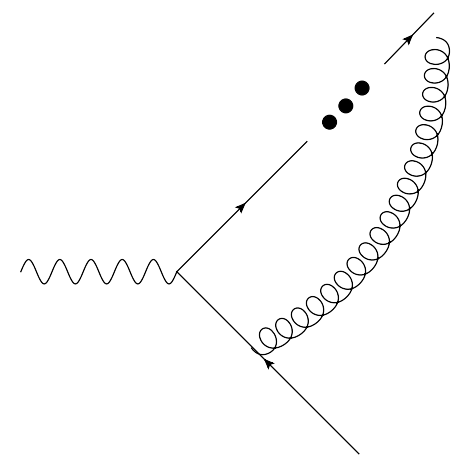

Figure 1: Example interaction, in which soft gluons may be emitted.

\section{Introduction}

It is well-known that scattering amplitudes in quantum field theory are beset by infrared divergences. Consider, for example, the interaction shown in figure 1, in which a vector boson splits into a quark pair. Either the final state quark or anti-quark may emit gluon radiation, and the Feynman rules in position space tell us that we must integrate over all positions of the emitted gluon. This integral goes out to infinity, and one may show from the behaviour of the integrand in four space-time dimensions that the region at infinity is associated with a (long-distance) divergence. We may think of such gluons as having an infinite Compton wavelength, and by the uncertainty principle this corresponds to the emission of gluons with zero momentum. Thus, these divergences are usually referred to as infrared (IR) divergences, and the gluons themselves as soft, to distinguish them from the hard emitting particles that emerge from the interaction. Such gluons may be real or virtual, and the above remarks make clear that IR divergences are a general feature of quantum field theories, including QED, QCD and quantum gravity.

Infrared singularities are important for a number of theoretical and phenomenological reasons. They are related to the structure of large logarithms in perturbation theory, which must be summed up to all orders in order to obtain sensible results for many collider observables (see [1, 2, 3, 4, 5] for a number of different approaches). Furthermore, there are a number of unproven conjectures regarding infrared divergences, such as the so-called dipole formula in QCD [6], which we will see in what follows. It has by now been well-established that scattering amplitudes factorise, such that they have the schematic form [7]

$$
A=H \cdot S \cdot \frac{\prod_{i} J_{i}}{\prod_{i} \mathscr{J}_{i}} .
$$

Here $H$ is the hard interaction, and is infrared finite; $S$ is the soft function, which collects all soft singularities; $J_{i}$ is a jet function, which collects hard collinear singularities associated with outgoing particle $i$. Finally, $\mathscr{J}_{i}$ is an eikonal jet function, which corrects for the double-counting of soft-collinear singularities associated with particle $i^{1}$. Furthermore, it is known in a variety of

\footnotetext{
9].

${ }^{1}$ In quantum gravity, jet functions are not present, as collinear singularities cancel upon combining all diagrams [8,
} 

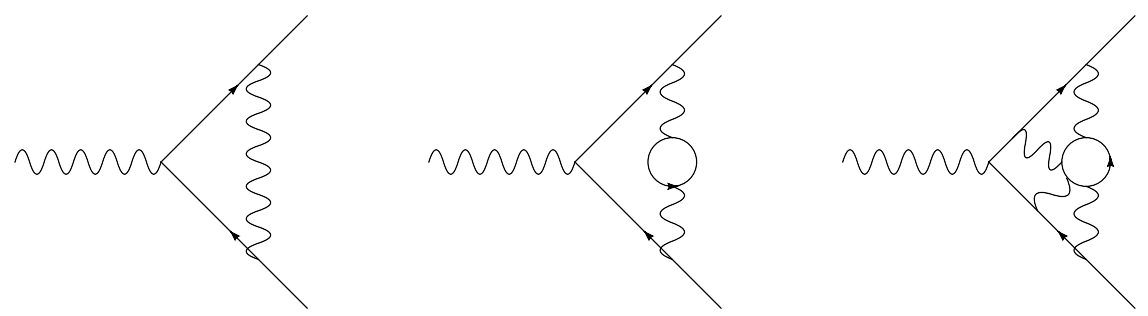

Figure 2: The first few webs in QED. Upon removing the external lines, one is left with connected subdiagrams.

theories that the soft function exponentiates, where the exponent may itself be given a Feynman diagram interpretation. That is, one may write

$$
S=\exp \left[\sum_{W} W\right],
$$

where $\{W\}$ is a set of special diagrams known as webs ${ }^{2}$. Their nature depends upon the theory.

\section{Webs in QED and QCD}

In QED, one may show that the exponent of the soft function contains only connected subdiagrams. By a subdiagram, we mean the graph that remains when the hard external lines have been removed. Examples of QED "webs" are shown in figure 2, and one can indeed see that they all correspond to connected subdiagrams. This result was originally derived using a combinatoric approach [12], and has recently been rederived in a more intuitive way using path integral methods and statistical physics techniques [5], which pave the way for examining more complicated cases of exponentiation e.g. multiparton scattering in QCD. Note that exponentiation is a very powerful result: it tells us that we can predict the structure of infrared divergences to all orders in perturbation theory. Successive powers of the coupling constant in the exponent sum up successive towers of IR singularities in the amplitude itself. Large logarithms that are associated with IR singularities can also then be predicted to all orders from soft exponentiation, which is how resummation works in practice.

The structure of soft exponentiation in QCD is more complicated due to the non-Abelian nature of the theory. Scattering amplitudes then have non-trivial colour structure. Furthermore, one must draw a distinction between processes in which only two coloured particles emerge from the hard interaction (e.g. Drell-Yan production of vector bosons, deep-inelastic scattering, $e^{+} e^{-} \rightarrow q \bar{q}$ ), and processes in which many hard coloured particles scatter. In the latter case, the scattering amplitude becomes a vector in the space of possible colour flows at the hard interaction vertex, and the soft

\footnotetext{
${ }^{2}$ This name was first introduced in the context of two-parton scattering in QCD [10], but we here adopt the more general terminology of [11].
} 


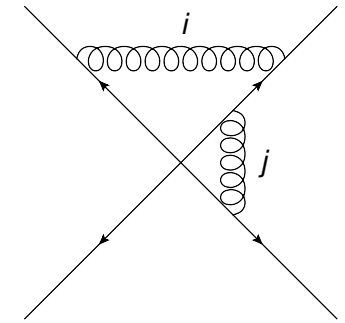

(a)

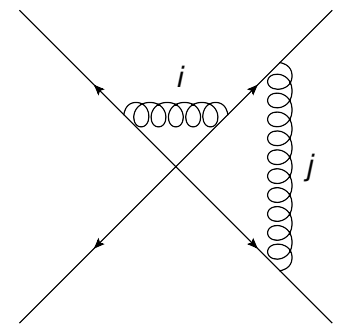

(b)

Figure 3: Diagrams contributing to the soft function at two loops, where solid and wavy lines denote hard and soft particles respectively.

function has a matrix structure in this space. Soft exponentiation in the two-particle case was first studied in [10], where it was found that the corresponding webs consist of single, irreducible subdiagrams (that is, subdiagrams where no gluon can be shrunk to the origin independently of any other gluon). The nature of webs in multiparton scattering has been studied only very recently [11, 13], due to the involved colour structure remarked upon above. The results are in marked contrast to the two-line case, and are best illustrated here by example.

Consider the pair of two-loop contributions to the soft function shown in figure 3 , where each diagram $D$ has a kinematic part $\mathscr{F}(D)$ and a colour factor $C(D)$. Although these diagrams are reducible (in the sense that one may shrink both gluons separately to the origin in each case), one may show that both of them contribute to the exponent of the soft function. Furthermore, each of them has a modified colour factor $\tilde{C}(D)$ in the exponent, which turns out to be a superposition of the usual colour factors $\{C(D)\}$ of both graphs. The contribution to the exponent from the pair can be written

$$
\left(\begin{array}{l}
\mathscr{F}(a) \\
\mathscr{F}(b)
\end{array}\right)^{T}\left(\begin{array}{l}
\tilde{C}(a) \\
\tilde{C}(b)
\end{array}\right)=\left(\begin{array}{l}
\mathscr{F}(a) \\
\mathscr{F}(b)
\end{array}\right)^{T} \frac{1}{2}\left(\begin{array}{rr}
1 & -1 \\
-1 & 1
\end{array}\right)\left(\begin{array}{l}
C(a) \\
C(b)
\end{array}\right) .
$$

We see that the pair of diagrams mixes under exponentiation, where this mixing can be described by a matrix acting on the vectors of kinematic and colour factors.

This structure is found to be quite general. Higher-loop diagrams form closed sets, where each set has elements related by gluon permutations on the external lines. It is argued in [11, 14] that each set should be considered as a single web, and its contribution to the exponent of the soft function is given by the generic form

$$
W=\sum_{D, D^{\prime} \in W} \mathscr{F}(D) R_{D D^{\prime}} C\left(D^{\prime}\right),
$$

where $R_{D D^{\prime}}$ is a web-mixing matrix. These matrices consist of constant numbers, and encode a huge amount of physics, namely how colour and kinematic information gets entangled in the soft limit to all orders in perturbation theory. An ongoing programme of work consists of classifying general properties of these matrices, and interpreting the corresponding physics. 
Some general properties are already known. For example, any row of any web mixing matrix must sum to zero. Also, all web mixing matrices are idempotent $\left(\mathbf{R}^{2}=\mathbf{R}\right)$, such that their eigenvalues can only be 1 or $0[11,15]$. A further conjecture involves a weighted sum of column entries [14]. A pure combinatoric expression for web mixing matrix elements has been given in [15], and may be related to order-preserving maps on partially ordered sets (posets) [16]. The latter are used in computer science applications, and thus progress in understanding webs can be made with or without any field theory knowledge.

For massless external particles, it is conjectured that the exponent of the soft function has a very simple form, involving colour and kinematic correlations between at most pairs of particles. This is known as the dipole formula [6], and possible corrections (at three loops and beyond) were further investigated in [17]. Recently, new constraints were found to emerge from the high energy (Regge) limit of scattering amplitudes [18], whose further implications were examined in [19]. Note that web mixing matrices should have something to say about the dipole formula: both webs and the dipole formula are concerned with the structure of the exponent of the soft function.

\section{IR singularities in gravity}

Infrared singularities in gravity were first examined in [8], and there has recently been a revival of interest aimed at describing gravitational physics using the same language as is used in nonabelian gauge theories $[9,20]$. It has now been firmly established that the exponent of the soft function in gravity is one-loop exact i.e. only one-loop diagrams occur, connecting all possible pairs of external particles. The relationship between QED / QCD and gravity was further explored in [21], using the radial coordinate space picture of [22]. This involves mapping the Wilson line operators which describe soft photon or graviton emissions from Minkowski space to Euclidean AdS space, where they become point charges whose potential energy represents the cusp anomalous dimension. The general potential energy for a spin- $n$ Wilson line is found to be

$$
\tilde{H}(\beta)=A_{1}\left(\frac{\sinh (n \beta)}{\sinh \beta}\right)+A_{2}\left(\frac{\cosh (n \beta)}{\sinh \beta}\right),
$$

where $\beta$ is the radial distance in the AdS space (equivalent to the cusp angle in Minkowski space). Equation (3.1) can be related to the known cusp anomalous dimensions in QED / QCD $(n=1)$ and gravity $(n=2)$. Furthermore, $n$ can be taken to be a continuous variable, and one thus sees that the soft limits of the two types of theory are related by a continuous deformation. This is an interesting novelty, that may have further implications.

Note that one-loop exactness of gravity implies that all IR singularities in this theory are dipole-like. This begs the question: could the QCD dipole formula have a gravitational origin? This has been further explored in [23], and the answer appears to be no. Nevertheless, intriguing connections between QCD and gravity exist, whose investigation is ongoing.

\section{References}

[1] G. F. Sterman, Nucl. Phys. B 281, 310 (1987). 
[2] S. Catani and L. Trentadue, Nucl. Phys. B 327, 323 (1989).

[3] G. P. Korchemsky and G. Marchesini, Phys. Lett. B 313, 433 (1993).

[4] T. Becher and M. Neubert, Phys. Rev. Lett. 97, 082001 (2006) [hep-ph/0605050].

[5] E. Laenen, G. Stavenga and C. D. White, JHEP 0903, 054 (2009) [arXiv:0811.2067 [hep-ph]].

[6] T. Becher and M. Neubert, Phys. Rev. Lett. 102, 162001 (2009) [arXiv:0901.0722 [hep-ph]];

T. Becher and M. Neubert, JHEP 0906, 081 (2009) [arXiv:0903.1126 [hep-ph]].

E. Gardi and L. Magnea, JHEP 0903, 079 (2009) [arXiv:0901.1091 [hep-ph]].

[7] J. C. Collins, Phys. Rev. D 22, 1478 (1980).
A. Sen, Phys. Rev. D 24, 3281 (1981).
G. P. Korchemsky, Phys. Lett. B 217, 330 (1989).
L. Magnea and G. F. Sterman, Phys. Rev. D 42, 4222 (1990).

[8] S. Weinberg, Phys. Rev. 140, B516 (1965).

[9] R. Akhoury, R. Saotome and G. Sterman, Phys. Rev. D 84, 104040 (2011) [arXiv:1109.0270 [hep-th]].

[10] J. G. M. Gatheral, Phys. Lett. B 133, 90 (1983).

J. Frenkel and J. C. Taylor, Nucl. Phys. B 246, 231 (1984).

[11] E. Gardi, E. Laenen, G. Stavenga and C. D. White, JHEP 1011, 155 (2010) [arXiv:1008.0098 [hep-ph]].

[12] D. R. Yennie, S. C. Frautschi and H. Suura, Annals Phys. 13, 379 (1961).

[13] A. Mitov, G. Sterman and I. Sung, Phys. Rev. D 82, 096010 (2010) [arXiv:1008.0099 [hep-ph]].

[14] E. Gardi, J. M. Smillie and C. D. White, JHEP 1109, 114 (2011) [arXiv:1108.1357 [hep-ph]].

[15] E. Gardi and C. D. White, JHEP 1103, 079 (2011) [arXiv:1102.0756 [hep-ph]].

[16] M. Dukes, E. Gardi, E. Steingrímsson and C. D. White, Submitted to Journal of Combinatoric Theory, Series A.

[17] L. J. Dixon, E. Gardi and L. Magnea, JHEP 1002, 081 (2010) [arXiv:0910.3653 [hep-ph]].

[18] V. Del Duca, C. Duhr, E. Gardi, L. Magnea and C. D. White, Phys. Rev. D 85, 071104 (2012) [arXiv:1108.5947 [hep-ph]].

V. Del Duca, C. Duhr, E. Gardi, L. Magnea and C. D. White, JHEP 1112, 021 (2011) [arXiv:1109.3581 [hep-ph]].

[19] L. Vernazza, PoS EPS -HEP2011, 284 (2011) [arXiv:1112.3375 [hep-ph]].

V. Ahrens, M. Neubert and L. Vernazza, JHEP 1209, 138 (2012) [arXiv:1208.4847 [hep-ph]].

[20] S. G. Naculich and H. J. Schnitzer, JHEP 1105, 087 (2011) [arXiv:1101.1524 [hep-th]].

C. D. White, JHEP 1105, 060 (2011) [arXiv:1103.2981 [hep-th]].

M. Beneke and G. Kirilin, JHEP 1209, 066 (2012) [arXiv:1207.4926 [hep-ph]].

[21] D. J. Miller and C. D. White, Phys. Rev. D 85, 104034 (2012) [arXiv:1201.2358 [hep-th]].

[22] Y. -T. Chien, M. D. Schwartz, D. Simmons-Duffin and I. W. Stewart, Phys. Rev. D 85, 045010 (2012) [arXiv:1109.6010 [hep-th]].

[23] S. Oxburgh and C. D. White, arXiv:1210.1110 [hep-th]. 